Proceedings

\title{
Design and Application of a Passive Acoustic Monitoring System in the Spanish Implementation of the Marine Strategy Framework Directive
}

\author{
Guillermo Lara ${ }^{1, *}$, Manuel Bou-Cabo ${ }^{2}$, Jose Antonio Esteban ${ }^{3}$, Victor Espinosa ${ }^{3}$ and Ramón \\ Miralles 1,* \\ 1 ITEAM - Universitat Politècnica de València \\ 2 Instituto Español de Oceanografía (IEO) \\ 3 IGIC - Universitat Politècnica de València \\ * Correspondence: guilamar@upv.es, rmiralle@dcom.upv.es, ITEAM-UPV, Cami de Vera s/n 46022 Valencia, Spain \\ + Presented at the 6th International Electronic Conference on Sensors and Applications, \\ 15-30 November 2019; Available online: https:/ / ecsa-6.sciforum.net/
}

Published: 14 November 2019

\begin{abstract}
A passive acoustic monitoring (PAM) device named SAMARUC has been developed to acquire underwater sounds following the specifications of the Monitoring Guidance for Underwater Noise in European Seas: Monitoring Guidance Specifications. Based on a Texas Instruments processor, an ultra-low power ADC was programmed to work at a sampling rate of $192 \mathrm{kHz}$ and adhoc electronics were designed allowing the processor's two microSD buses to be used, thereby increasing the storage capacity. Many other software and hardware enhancements were implemented, such as the new low latency file system, the construction of NITUFF anodized aluminium housing and ringed buoys. With the resulting application, data obtained by the SAMARUC at El Gorguel (Cartagena, Spain) in 2018 were compared to a theoretical underwater noise map created using AIS data. This was done following the Descriptor D11.2 by means of the ambient noise level indicators at two one-third-octave frequency bands $(63 \mathrm{~Hz}$ and $125 \mathrm{~Hz})$, mainly related to marine traffic and noise pollution. The conjunction between the acquisition of underwater acoustic data and the development of a numerical propagation model was found to be highly recommendable to estimate the ambient continuous noise level when validating the acquired data as well as when correcting the prediction provided by the model.
\end{abstract}

Keywords: underwater acoustics; numerical methods; passive acoustic monitoring

\section{Introduction}

Currently, one of the hot topics in underwater acoustics concerns the implementation by European Union member states of monitoring programmes that ensure the assessment of underwater noise levels due to anthropogenic sources (of an impulsive or continuous nature) and their effects on marine biota. These monitoring programmes must apply standarized methods to assess the good environmental status (GES) in European seas. For example, the Monitoring Guidance for Underwater Noise in European Seas: Monitoring Guidance Specifications [1] established by TGNoise recommends high performance technical specifications for the monitoring devices (for instance, very low self-noise), which are not met by many commercial devices.

Moreover, in order to carry out a correct assessment of underwater noise, experimental measurements and numerical sound propagation simulations that consider the marine traffic in specific zones are 
necessary. However, simulations may leave a lot of uncertainties with respect to the source noise level assigned to every ship, or with respect to water column properties like salinity and temperature, or even as regards marine ambient noise due to weather conditions. Experimental measurements, on the other hand, can act not only as a real assessment of underwater noise in a specific location, but are also an essential tool to validate the simulated sound map. Hence, the reason for this work goes far beyond the scope of the research and development process of constructing a passive acoustic monitoring (PAM) device.

This paper describes the conceptualization, design and implementation of a PAM device called SAMARUC that allows underwater audio acquisition according to the specifications established by the Monitoring Guidance for Underwater Noise in European Seas: Monitoring Guidance Specifications [1]. It goes on to describe an application as an example of the work currently being carried out by the joint IEO-UPV unit (Instituto Español de Oceanografía and Universitat Politècnica de València), the body in charge of implementing the Marine Strategy for underwater noise in Spain. From the data acquired by SAMARUC, two indicators associated with ambient noise are obtained in this work in order to finally compare them with those obtained through a numerical method based on ray theory approximation using the RANDI model [2] to parameterize the source noise level of each ship in the region studied, identified by their AIS data.

\section{Materials and Methods}

\subsection{SAMARUC as Passive Acoustic Monitoring Device}

The SAMARUC autonomous system focuses on providing high bandwidth submarine audio recordings (192 kHz sampling rate) coupled with accurate sound pressure measurements. The system's main component is a general-purpose ultra-low power consumption digital signal processing board (TMS320C5535). The board is set up in DMA Ping-Pong mode so the system records acoustic data without sample loss while storing that data in the microSD cards.

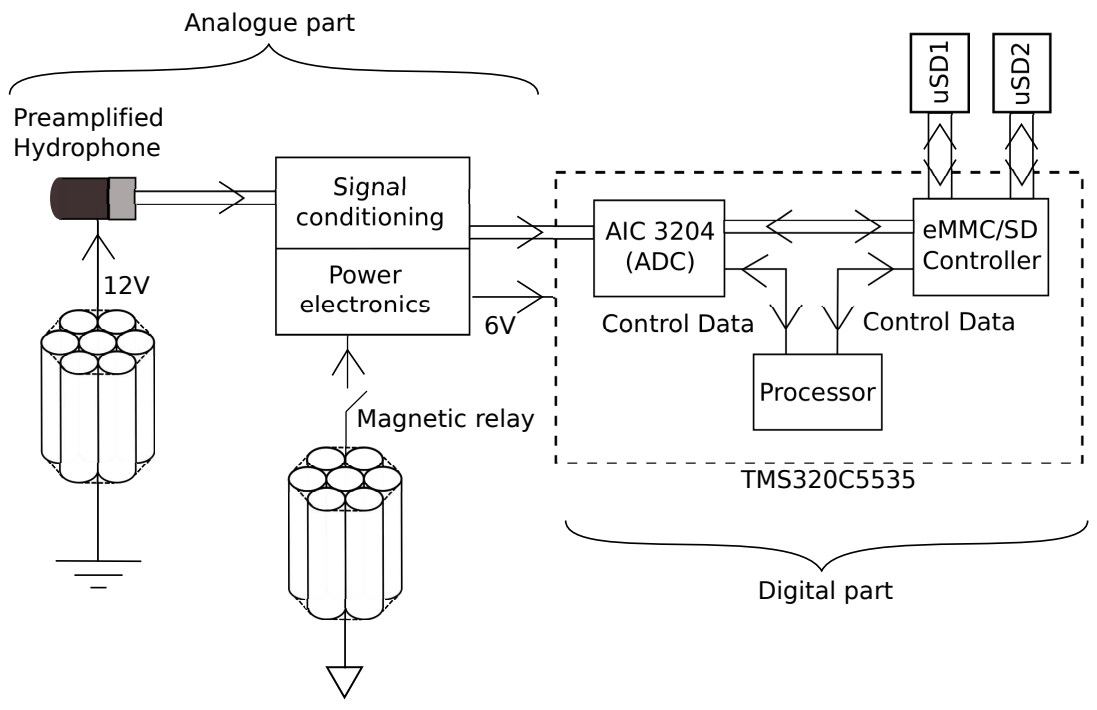

Figure 1. Block diagram of the SAMARUC system

Figure 1 shows how the electronics used work in a block diagram. One can see how the TMS320C5535 processor controls the digital data from the analogue-to-digital converter (ADC) AIC3204 so that it arrives 
through the data bus to the microSD cards. To enhance the signal-to-noise ratio, the grounds of the power supply circuit and the conditioning circuit have been isolated.

Acquisition of the audio signal via the AIC3204 is set at a sampling rate of $192 \mathrm{kHz}$ at 16 bits, with the advantage of being able to add a gain to amplify the signal if it is necessary to increase the sensitivity of the selected hydrophone depending on the deployment category (A to establish the ground truth in quiet areas, and B for noisy placements [1]). In any case, a system sensitivity (hydrophone + AIC3204 gain) of between -165 and $-185 \mathrm{~dB}$ re $1 \mathrm{~V} / \mathrm{uPa}$ is recommended [1]. Table 1 sums up the main specifications of the SAMARUC, many of them obtained by means of the calibration carried out in the IEO-UPV facilities located in the port of Gandía (Valencia, Spain) following the recommendations given in [1]. The choice of hydrophone is one of the most important matters in order to achieve the desired sensitivity.

Table 1. Specifications

\begin{tabular}{cc}
\hline Sensitivity of the preamplified hydrophone & $\mathbf{- 1 6 7} \mathbf{~ d B}$ re 1V/uPa (Cetacean Research C57) \\
\hline \# batteries used (with/without preamplification) & $36 / 28$ (type D) \\
AIC3204 programmable gain & $0-30 \mathrm{~dB}$ \\
Storage capacity & Currenty: (1+1) Tbytes \\
Sampling rate & $192 \mathrm{kHz}$ \\
System bandwidth $\pm \mathbf{3 d B}$ & $10 \mathrm{~Hz}-96 \mathrm{kHz}$ \\
Maximum depth & $500 \mathrm{~m}$. (Housing and buoys) \\
Autonomy & Up to 4 months \\
\hline
\end{tabular}

\subsubsection{Specific Electronics}

A dedicated electronic board was designed with the aim of adding a second microSD card to double the available storage capacity. In addition, this electronic design allows the hydrophone (audio) power circuit to be isolated from the evaluation board's power supply by adding two independent grounds. This reduces the interferences from the processor's electronics on the low-level hydrophone signal, increasing the signal-to-noise ratio.

\subsubsection{Fyle System}

The standard libraries of the TMS320C5535 evaluation board allowed for a maximum storage capacity of 32 Gbytes (FAT32 file system). It was necessary to design a new low latency file system with a new set of access functions to read and write, while being able to use all of the microSD card's storage capacity.

\subsubsection{Housing and Battery Pack}

A pressure case was built from a tube of 6082 aluminium anodized with NITUFF, measuring $12 \times 70$ $\mathrm{cm}$ (diameter $\times$ length). This material was chosen according to relevant factors such as durability (the anodized NITUFF is used in military marine applications) and being lightweight. Moreover, in order to maximize the energy density in the cylindrical housing, a hexagonal layout of D-cell batteries was created. The battery pack for the main electronics is composed of $7 \times 4$ D-type batteries. This battery pack allows for deployments of up to 4 months and $500 \mathrm{~m}$ of depth.

\subsubsection{Ringed Buoys and Hydrophone Protection}

Ringed buoys were used to enable handling of the device and avoid acoustic shadows, providing the system with the necessary buoyancy and allowing deployments down to depths of $500 \mathrm{~m}$. In addition, a 
removable cage was designed to keep the hydrophone in a vertical position, isolate it from vibrations and protect it from shocks. Table 2 shows a brief summary of the integrated functions.

Table 2. Summary

\begin{tabular}{cc}
\hline Element & Functionality \\
\hline File system & Increase the system storage capacity, previously limited by the FAT32 system file (32 Gbytes) \\
Specific electronics & Isolate the grounds and use a second microSD card. Storage capacity x 2 \\
Housing & 6082 aluminium, widely used in marine applications \\
Battery block & Maximize energy density according to the tube dimensions \\
Flotation buoys & Ease of handling and avoidance of acoustic shadows \\
Protection cage & Hydrophone protection \\
\hline
\end{tabular}

\subsection{Data Acquistion and Study Area}

Data were collected using the previously described SAMARUC device from 16 May 2018 to 30 July 2018 in the shallow waters of El Gorguel ( $37^{\circ} 33^{\prime} 42^{\prime \prime}$ N, $0^{\circ} 53^{\prime} 14^{\prime \prime}$ E) on the south-east coast of the Mediterranean Sea (Cartagena, Spain). This location represents the marine traffic due to Cartagena's port. The seabed in this area is not uniform and consists basically of mud.

The minimum distance between the device and the vessels' permitted transit area was approximately $150 \mathrm{~m}$ (category B [1]). The device was placed $10 \mathrm{~m}$ from the seabed (reducing its acoustic influence) at a depth of $50 \mathrm{~m}$ using a $70 \mathrm{~kg}$ anchoring weight. The sampling frequency was set to $192 \mathrm{kHz}$ with a resolution of 16 bits and a device sensitivity set to $-165 \mathrm{~dB}$ re $1 \mathrm{~V} / \mathrm{uPa}(-167 \mathrm{~dB}$ re $1 \mathrm{~V} / \mathrm{uPa}$ hydrophone sensitivity $+2 \mathrm{~dB}$ of AIC3204 gain). The SAMARUC was deployed for two months, setting an operating cycle of 5 min ON and 10 min OFF.

\subsection{Numerical Modelling of Sound Propagation}

There are different kinds of numerical approximations to solve the propagation of underwater sound in a marine environment (e.g. integration of parabolic equation, ray theory, normal modes, etc.) [3]. Each method has its pros and cons depending on the acoustic frequency considered, seabed depth and sound speed profiles in the water column.

In this case, we chose ray theory approximation. A public code called BELLHOP [4] was applied because it needs less computing resources than other numerical methods like the integration of the parabolic equation [3]. Theoretically, ray approximation is more suited to high frequency propagation (in the order of tens of $\mathrm{kHz}$ ) but one of the aims of this study was to check if this method provides enough accuracy if both the receiver and emitter are at a distance of at least half a wavelength of the propagated signal from the seabed and sea surface [5].

Our computations consider the speed of sound calculated for the water column range using Argo profiler data: temperature, salinity and depth/pressure available on the EMODnet portal website [6] for the same month as the marine traffic data obtained from the Automatic Identification System (AIS) database. The velocity of sound vs depth was calculated by applying the nine-term Mackenzie equation [7] due to its validity range, considering temperature between $2^{\circ} \mathrm{C}$ and $30^{\circ} \mathrm{C}$, salinity of $25-40 \mathrm{ppt}$ (parts per thousand) and a depth of between $0 \mathrm{~m}$ to $8000 \mathrm{~m}$. As regards the bathymetric data, the seabed was defined using public data from the General Bathymetric Chart of the Oceans [8].

The RANDI model [2] was used to parameterize the source level of each ship considered in the computed AIS data. The reason for this choice is that it is accepted that the validity range in terms of frequency is defined between $28.4 \mathrm{~Hz}-191.6 \mathrm{~Hz}$, which is suitable for our study ( $63 \mathrm{~Hz}$ and $125 \mathrm{~Hz})$. The 
mean value of the Sound Pressure Level (SPL) over the location was calculated considering the AIS data of vessels that have non-zero speed over ground.

\section{Results and Discussion}

The results obtained considering ray theory approximation are summarized in the sound maps of daily SPL $\mathrm{dB}$ re $1 \mathrm{~V} / \mathrm{uPa}$ at a depth of $50 \mathrm{~m}$. Figure 2 shows the daily sound map of the region studied after computing AIS data in the propagation model at frequencies of $63 \mathrm{~Hz}$ and $125 \mathrm{~Hz}$ on the day 16/05/2018. In addition, it is important to remark that both sound maps were calculated at a depth of $50 \mathrm{~m}$, which is the depth where the SAMARUC device was installed (marked with a black star).
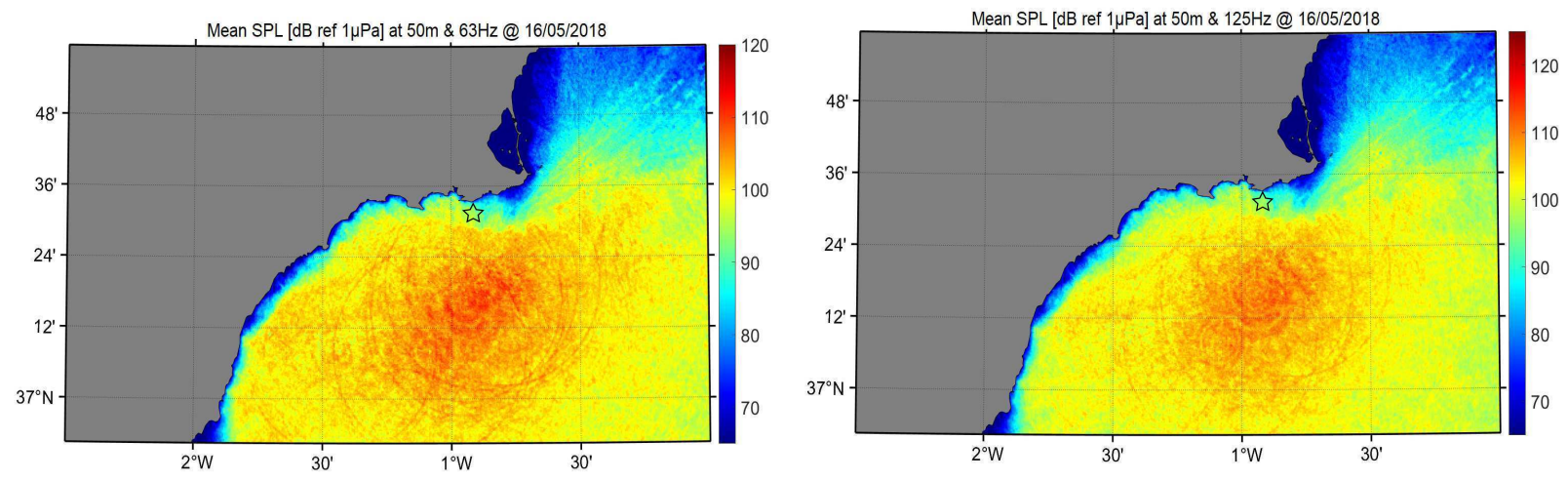

Figure 2. Sound map on 16/05/2018 at (a) $63 \mathrm{~Hz}$ and (b) $125 \mathrm{~Hz} \mathrm{~Hz}$ calculated over the region studied

It should also be taken into account that values of latitude greater than 36 degrees may be unrealistic, since AIS data were not available at those latitudes. Daily sound maps of Figure 2 were calculated using four samples of AIS data for certain hours of the day $(6: 00 \mathrm{~h}-7: 00 \mathrm{~h}, 14: 00 \mathrm{~h}-15: 00 \mathrm{~h}, 19: 00 \mathrm{~h}-20: 00 \mathrm{~h}$ and 23:00 $\mathrm{h}-00: 00 \mathrm{~h}$ ) at the same location of the SAMARUC, obtaining a daily mean value of pressure within a standard deviation interval (see Table 3).

Table 3. dB SPL re 1V/uPa comparison for $63 \mathrm{~Hz}$ and $125 \mathrm{~Hz}$ on 16/05/2018

\begin{tabular}{c|ccccc}
\hline 16 May 2018 & \multicolumn{5}{|c}{$\mathbf{6 3 ~ H z}$} \\
\hline Hours & $6: 00 \mathrm{~h}-7: 00 \mathrm{~h}$ & $14: 00 \mathrm{~h}-15: 00 \mathrm{~h}$ & $19: 00 \mathrm{~h}-20: 00 \mathrm{~h}$ & $23: 00 \mathrm{~h}-00: 00 \mathrm{~h}$ & Average \\
\hline SAMARUC & $78 \pm 13$ & $85.3 \pm 0.5$ & $79.7 \pm 0.7$ & $83.1 \pm 1.2$ & $82 \pm 6$ \\
\hline Ray model & $81 \pm 3$ & $91 \pm 5$ & $78 \pm 3$ & $84 \pm 2$ & $83 \pm 5$ \\
\hline & \multicolumn{7}{|c}{$\mathbf{1 2 5} \mathbf{H z}$} \\
\hline Hours & $6: 00 \mathrm{~h}-7: 00 \mathrm{~h}$ & $14: 00 \mathrm{~h}-15: 00 \mathrm{~h}$ & $19: 00 \mathrm{~h}-20: 00 \mathrm{~h}$ & $23: 00 \mathrm{~h}-00: 00 \mathrm{~h}$ & Average \\
\hline SAMARUC & $85 \pm 4$ & $90 \pm 2$ & $83.8 \pm 0.3$ & $81 \pm 2$ & $85 \pm 9$ \\
\hline Ray model & $83 \pm 4$ & $93 \pm 3$ & $81.4 \pm 1.1$ & $81 \pm 4$ & $85 \pm 6$ \\
\hline
\end{tabular}

Going back to real data obtained by SAMARUC, we calculated the average one-third-octave band (also in SPL dB re $1 \mathrm{~V} / \mathrm{uPa}$ for $63 \mathrm{~Hz}$ and $125 \mathrm{~Hz}$ ) for an integration interval of one hour at the same timestamp hours of the modelling calculations. The automated SPL analyses were performed using Samaruc Laboratory software (ITEAM-UPV, Valencia, Spain). As Table 3 shows, both the one-third-octave 
band indicators from the real and theoretical data were calculated following the framework of Descriptor 11 [9] of the European MSFD.

The results show a good agreement between experimental and theoretical calculations, verifying not only that the device worked properly and the numerical simulations were valid, but also the calibration of the system performed at IEO-UPV's facilities, an aspect not easy to achieve due to the low frequency range considered.

\section{Conclusions}

In this work, the whole process of underwater noise assessment has been performed, from theoretical sound map simulation to validation by means of experimental measurements. This has been done with the aim of calculating the underwater sound pressure level associated with a continuous noise coming from anthropogenic activities (marine traffic). To achieve this goal, a PAM system has been specifically designed meeting all the specifications imposed by the technical guidelines proposed by [1]. The system designed is modular and can be programmed to measure different kinds of categories, from quiet to noisy ones.

Several features will be improved in future works, such as researching other acoustic metrics, looking deeper into the assessment of acoustic noise effects on marine biota and improving some characteristics of the PAM device (deployment depth, higher bandwidth, new sensors, etc).

\section{Patents}

All SAMARUC Software is protected by UPV copyright.

\section{Abbreviations}

The following abbreviations are used in this manuscript:

PAM Passive Acoustic Monitoring

MSFD Marine Strategy Framework Directive

GES Good Environmental Status

ADC Analog to Digital Converter

SPL Sound Pressure Level

\section{References}

1. Dekeling, R.P.A.; Tasker, M.L.; Van der Graaf, A.J.; Ainslie, M.A.; Andersson, M.H.; André, J.F.; Borsani, K.; Brensing, M.; Castellote, D.; Cronin, J.; and et.al Monitoring Guidance for Underwater noise in European Seas: Monitoring Guidance specifications, Sci. Policy Rep., ISSN 1831-9424.

2. Breeding, J.E.; Plug, L.A.; Bradley, E.L.; Walrod, M.H. and McBride, W. Research Ambient Noise Directionality (RANDI) 3.1 - Physics description, Technical Report NRL/FR/7176-95-9628, Naval Research Laboratory 1996

3. Etter, P.C. Underwater Acoustic Modeling and Simulation. CRC Press—5th Edition 2018, ISBN-10: 1138054925

4. Porter, M.B. and Liu, Y. Finite element Ray Tracing. J. Theor. Comput. Acous. 1994, 2, 947-956.

5. Hovem, J.M. Ray Trace Modeling of Underwater Sound Propagation. In Modeling and Measurement Methods for Acoustic Waves and for Acoustic Microdevices 2013 ISBN: 978-953-51-1189-4.

6. https://www.emodnet-physics.eu/Map/. Last connection on 09/09/2019.

7. Mackenzie, K.V. Nine-term equation for the sound speed in the oceans. J. Acoust. Soc. Am. 1981, 70, 807-812.

8. https://www.gebco.net/data_and_products/gridded_bathymetry_data/. Last connection on 09/09/2019.

9. Commission Decision (EU) 2017/848 Official Journal of the European Union 2017. 
(C) 2019 by the authors. Licensee MDPI, Basel, Switzerland. This article is an open access article distributed under the terms and conditions of the Creative Commons Attribution (CC BY) license (http:/ / creativecommons.org/licenses/by/4.0/). 